\title{
Reduced Complexity Polynomial Expansion Approximation to MMSE-DFE
}

\author{
V.K. Varma Gottumukkala, Hlaing Minn, Naofal Al-Dhahir \\ Department of Electrical Engineering \\ University of Texas at Dallas \\ Richardson, Texas 75080 \\ Email: \{vkg071000, hlaing.minn, aldhahir $\} @$ utdallas.edu
}

\begin{abstract}
In this paper, we investigate polynomial expansion approximation to reduce matrix inversion complexity as encountered in the design of the Minimum Mean Squared Error Decision Feedback Equalizer (MMSE-DFE). The scaling factor needed in this polynomial expansion is optimized for a fixed polynomial approximation order so that the received Signal to Interference plus Noise Ratio (SINR) is maximized. The BER performance of the reduced-complexity MMSE-DFE is comparable to that of the direct matrix inversion based MMSE-DFE and outperforms earlier approaches reported in the literature.
\end{abstract}

\section{INTRODUCTION}

Channels with Inter Symbol Interference (ISI) are commonly encountered in broadband communication systems. Several equalization structures have been proposed to mitigate ISI effects [1]. The MMSE-DFE is a popular equalization structure as it achieves a practical tradeoff between performance and complexity [2]. However, the MMSE-DFE still requires matrix inversion whose computational cost is high. In general, this complexity is cubic in the total number of feedforward and feedback taps although it can be reduced by an order of magnitude in some special cases (e.g. when the number of feedback taps is set equal to the channel memory [3]. In the literature, some methods ([4], [5], [6]) have been proposed to reduce the cubic computational complexity involved in the matrix inversion for the MMSE-DFE in the general case. These methods involve approximating the matrix inversion with a polynomial expansion. However, the parameters in the polynomial expansion have to be optimized. Specifically, the scaling factor in the polynomial expansion has to be suitably set so that the resulting equalizers perform as desired. References [5] and [6] used two different approaches for setting the polynomial expansion scaling parameters. In [4], a different method was used to find the scaling parameter, namely, by maximizing a system performance criterion. However, the parameters for the polynomial expansion were derived for the simpler case of the Minimum Mean Squared Error - Linear Equalizer (MMSE-LE).

In this paper, the polynomial expansion parameters are found by using a similar criterion as in [4]. However, these parameters are derived for the more sophisticated MMSEDFE equalizer. When there is no feedback filter (in which case, the MMSE-DFE simplifies to the simpler MMSE-LE), our results simplify to the results obtained in [4] for the MMSE-LE. As in [4], the received Signal to Interference plus Noise Ratio is used to optimize the polynomial expansion parameters. In particular, the parameters have been optimized with respect to a fixed polynomial order. We demonstrate through simulations that our proposed equalizer outperforms earlier approaches in terms of bit error rate (BER). From the simulation results, it is also found that the polynomial approximation technique is more attractive for the MMSEDFE case as it achieves high performance even with a lowerorder polynomial approximation compared to the higher-order polynomial approximations needed for the MMSE-LE case studied in [4].

The remainder of this paper is organized as follows: Section II describes the system model. Section III introduces the polynomial approximation to matrix inversion in general. In section IV, we describe the polynomial approximation as applied to the MMSE-DFE and consider the first order approximation in particular. Simulation results and discussions are presented in Section V and finally we conclude in Section VI. The following notation is used throughout this paper. 1) vector - bold font, small letter, 2) matrix - bold font, capital letter, 3) scalar - not in bold, 4) $(.)^{H}$ and $(.)^{T}$ - conjugate transpose and transpose respectively, 5) $0_{p \times q}$ - all zeros matrix of size $p \times q 6) \mathbf{e}_{p}$ - column vector of appropriate length, consisting of all zeros and a 1 in the $p$ th location, 7) $\operatorname{tr}(\mathbf{A})$ - trace of matrix $\mathbf{A}$.

\section{SySTEM ModeL}

Consider the discrete-time ISI channel model

$$
\mathbf{y}_{k}=\mathbf{H} \mathbf{x}_{k}+\mathbf{z}_{k}
$$

where $\mathbf{y}_{k}=\left[y_{k}, y_{k-1}, \ldots, y_{k-\left(N_{f}-1\right)}\right]^{T}$ is the column vector of $N_{f}$ output symbols, $\mathbf{H}$ is the $N_{f} \times\left(N_{f}+\nu\right)$ channel convolution matrix ( $\nu$ is the channel memory order), $\mathbf{x}_{k}=$ $\left[x_{k}, x_{k-1}, x_{k-2}, \ldots, x_{k-\Delta}, x_{k-\Delta-1}, \ldots, x_{k-\Delta-N_{b}}, \ldots\right.$ $\left.\ldots, x_{k-\left(N_{f}+\nu-1\right)}\right]^{T}$ is the column vector of $N_{f}+\nu$ input symbols, $\mathbf{z}_{k}=\left[z_{k}, z_{k-1}, \ldots, z_{k-\left(N_{f}-1\right)}\right]^{T}$, is the noise column vector consisting of $N_{f}$ elements. The noise elements are iid, Gaussian with zero mean and variance $\sigma_{z}^{2}$. The MMSE-DFE processes this block of $N_{f}$ output symbols $\mathbf{y}_{k}$ and $N_{b}$ input symbols $\mathbf{x}_{k-\Delta-N_{b}}^{k-\Delta-1} \triangleq\left[x_{k-\Delta-1}, x_{k-\Delta-2} \ldots, x_{k-\Delta-N_{b}}\right]^{T}$ and 
makes a decision on the element $x_{k-\Delta} . \Delta$ is the decision delay that is inherent in the functioning of the MMSE-DFE. The MMSE-DFE consists of a feedforward filter (FFF) that has $N_{f}$ taps: $\mathbf{w}=\left[w_{1}, w_{2}, \ldots, w_{N_{f}}\right]^{T}$ and a feedback filter $(\mathrm{FBF})$ that has $N_{b}$ taps: $\mathbf{b}=\left[b_{1}, b_{2}, \ldots, b_{N_{b}}\right]^{T}$. Let $\tilde{\mathbf{w}} \triangleq\left[\begin{array}{c}\mathbf{w} \\ \mathbf{b}\end{array}\right]$ be the column vector consisting of the elements of both the FFF and FBF filters. Then, we have for the MMSE-DFE [7]

$$
\tilde{\mathbf{w}}^{H}=E_{x} \tilde{\mathbf{h}}_{\Delta}^{H} \mathbf{R}_{\tilde{\mathbf{y}}_{k}}^{-1}
$$

where

$$
\begin{gathered}
\tilde{\mathbf{y}}_{k}=\left[\begin{array}{c}
\mathbf{y}_{k} \\
\mathbf{x}_{k-\Delta-N_{b}}^{k-\Delta-1}
\end{array}\right], \\
\tilde{\mathbf{h}}_{\Delta}^{H}=\left[\mathbf{h}_{\Delta}^{H}, \mathbf{0}_{1 \times N_{b}}\right]
\end{gathered}
$$

and $\mathbf{h}_{\Delta}$ is the $(\Delta+1)$ th column vector of $\mathbf{H}, E_{x}$ is the average energy of each input symbol, $\mathbf{R}_{\tilde{\mathbf{y}}_{k}}$ is the $\left(N_{f}+N_{b}\right) \times\left(N_{f}+N_{b}\right)$ covariance matrix of $\tilde{\mathbf{y}}_{k}$ given by

$$
\mathbf{R}_{\tilde{\mathbf{y}}_{k}}=\left[\begin{array}{cc}
E_{x} \mathbf{H} \mathbf{H}^{H}+\frac{\mathbf{R}_{\mathbf{z z}}}{{ }_{\mathrm{SNR}}} & E_{x} \mathbf{H} \mathbf{J}_{\Delta} \\
E_{x} \mathbf{J}_{\Delta}^{H} \mathbf{H}^{H} & E_{x} \mathbf{I}_{N_{b}}
\end{array}\right]
$$

where, $\mathrm{SNR} \triangleq \frac{E_{x}}{\sigma^{2}}$ and $\mathbf{J}_{\Delta}$ is an $\left(N_{f}+\nu\right) \times N_{b}$ matrix of 0 's and 1 's with 0 's in the upper $\Delta+1$ rows and an identity matrix of dimension $\min \left\{N_{b}, N_{f}+\nu-\Delta-1\right\}$ with 0's to its right (when $N_{b}>N_{f}+\nu-\Delta-1$ ) or zeros below it (when $N_{b}<N_{f}+\nu-\Delta-1$ ) or no further zeros and fitting exactly (when $N_{b}=N_{f}+\nu-\Delta-1$ ).

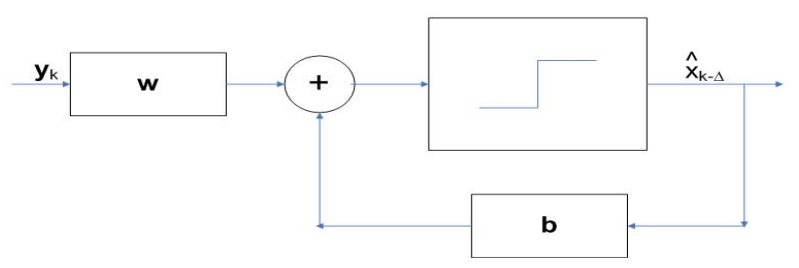

Fig. 1. Block Diagram of the MMSE-DFE with the FFF and FBF.

\section{POLYNOMIAL APPROXIMATION FOR MATRIX INVERSION}

Let the eigenvalues of the matrix $\mathbf{R}_{\tilde{\mathbf{y}}_{k}}$ be denoted by $\lambda_{i}, \quad i=1, \ldots,\left(N_{f}+N_{b}\right)$. The polynomial approximation to matrix inversion $\mathbf{R}_{\tilde{\mathbf{y}}_{k}}^{-1}$ consists of a scaling factor $\psi$ which is determined such that $\left|1-\psi \lambda_{i}\right|<1, i=1, \ldots, N_{f}+N_{b}$. If the above condition is satisfied, then $\mathbf{R}_{\tilde{\mathbf{y}}_{k}}^{-1}$ can be approximated with the following series expansion

$$
\mathbf{R}_{\tilde{\mathbf{y}}_{k}}^{-1}=\psi \sum_{i=0}^{\infty}\left(\mathbf{I}_{N_{f}+I_{N_{b}}}-\psi \mathbf{R}_{\tilde{\mathbf{y}}_{k}}\right)^{i}
$$

This result is well known and has been used in [4], [5], [6] and [8]. In particular, by considering the $N$ th order series expansion with scaling factor $\psi(N)$ for $\mathbf{R}_{\tilde{\mathbf{y}}_{k}}^{-1}$, we get,

$$
\begin{array}{r}
\tilde{\mathbf{w}}_{(N)}^{H}=E_{x} \tilde{\mathbf{h}}_{\Delta}^{H} \psi(N)\left[\mathbf{I}_{N_{f}+N_{b}}+\left(\mathbf{I}_{N_{f}+N_{b}}-\psi(N) \mathbf{R}_{\tilde{\mathbf{y}}_{k}}\right)\right. \\
+\left(\mathbf{I}_{N_{f}+N_{b}}-\psi(N) \mathbf{R}_{\tilde{\mathbf{y}}_{k}}\right)^{2}+\ldots
\end{array}
$$

$$
\left.+\ldots+\left(\mathbf{I}_{N_{f}+N_{b}}-\psi(N) \mathbf{R}_{\tilde{\mathbf{y}}_{k}}\right)^{N}\right]
$$

When $N=0$, this filter becomes the matched filter. On the other hand, as $N \rightarrow \infty$ this filter converges to the MMSE-DFE. This follows from the fact that the polynomial approximation to the matrix inverse converges to the direct matrix inverse as the order of the polynomial goes to infinity [8]. From computational simplicity point of view, it is desirable to keep $N$ as low as possible while still maintaining sufficient performance (since higher order $N$ leads to better approximation to the MMSE-DFE with direct matrix inversion, resulting in better performance).

\section{POLYNOMIAL APPROXIMATION TO MATRIX INVERSION IN MMSE-DFE EQUALIZER}

Let the minimum and maximum eigenvalues of $\mathbf{R}_{\tilde{\mathbf{y}}_{k}}$ be denoted by $\lambda_{\min }$ and $\lambda_{\max }$, respectively. A simple analysis as shown in [5] tells us that convergence of $\tilde{\mathbf{w}}_{(N)}^{H}$ to $\tilde{\mathbf{w}}^{\mathbf{H}}$ is ensured if $\psi$ is chosen in the range $0<\psi<\frac{2}{\lambda_{\max }}$. [5] and [6] have proposed different approaches for selecting the scaling parameter $\psi$. In [5] the scaling parameter was set as $\psi=\frac{2}{\operatorname{tr}(\Sigma)}$ while in [6] the scaling parameter was taken as $\psi=\frac{2}{\lambda_{\min }+\lambda_{\max }}$. Both these methods ensure that $\psi$ lies in the required range $0<\psi<\frac{2}{\lambda_{\max }}$. The method used in [6] ensured fastest convergence rate when the two extreme modes $\left|1-\psi \lambda_{\min }\right|$ and $\left|1-\psi \lambda_{\max }\right|$ were identical [6], [9]. However, when there is a large spread of eigenvalues, a higher order $N$ is needed to maintain good performance. The authors in [4] have proposed an alternative method for such cases, and the scaling parameter was optimized with respect to a fixed order. The optimization criterion is the signal to interference and noise power ratio (SINR) at the equalizer output. The authors in [4] have performed the optimization for the simpler case of MMSE-LE. Following along similar lines, here we introduce the polynomial expansion approximation for the more sophisticated MMSE-DFE and perform optimization with respect to a fixed order. More explicitly, we consider the following first-order polynomial expansion approximation to the MMSE-DFE

$$
\tilde{\mathbf{w}}_{(1)}^{H}=E_{x} \tilde{\mathbf{h}}_{\Delta}^{H} \psi(1)\left[\mathbf{I}_{N_{f}}+\left(\mathbf{I}_{N_{f}}-\psi(1) \mathbf{R}_{\tilde{\mathbf{y}}_{k}}\right)\right] .
$$

By considering the expression for SINR at the equalizer output, the optimum scaling coefficient $\psi(1)$ is found by solving

$$
[\psi(1)]_{\mathrm{opt}}=\arg _{\psi(1)} \max \operatorname{SINR}
$$

where

$$
\operatorname{SINR}=\frac{E_{x} \tilde{\mathbf{w}}^{H} \tilde{\mathbf{h}}_{\Delta} \tilde{\mathbf{h}}_{\Delta}^{H} \tilde{\mathbf{w}}}{\tilde{\mathbf{w}}^{H} \mathbf{R}_{\tilde{\mathbf{y}}_{k}} \tilde{\mathbf{w}}-E_{x} \tilde{\mathbf{w}}^{H} \tilde{\mathbf{h}}_{\Delta} \tilde{\mathbf{h}}_{\Delta}^{H} \tilde{\mathbf{w}}}
$$

or

$$
[\psi(1)]_{\mathrm{opt}}=\arg _{\psi(1)} \max \left(\frac{\left\|\tilde{\mathbf{w}}_{(1)}^{H} \tilde{\mathbf{h}}_{\Delta}\right\|^{2}}{\tilde{\mathbf{w}}_{(1)}^{H} \mathbf{R}_{\tilde{\mathbf{y}}_{\mathbf{k}}} \tilde{\mathbf{w}}_{(1)}}\right) .
$$


The closed-form solution for $\psi(1)$ is obtained as

$$
\psi(1)=2 \frac{\boldsymbol{\beta}^{H} \boldsymbol{\gamma} \boldsymbol{\beta}}{\boldsymbol{\beta}^{H} \boldsymbol{\gamma} \mathbf{R}_{\tilde{\mathbf{y}}_{k}} \boldsymbol{\beta}}
$$

where $\boldsymbol{\beta}^{H}=\tilde{\mathbf{h}}_{\Delta}^{H} \mathbf{R}_{\tilde{\mathbf{y}}_{k}}$ and $\boldsymbol{\gamma}=\left\|\tilde{\mathbf{h}}_{\Delta}\right\|^{2} \mathbf{I}_{N_{f}+N_{b}}-\tilde{\mathbf{h}}_{\Delta} \tilde{\mathbf{h}}_{\Delta}^{H}$. The details of the derivation are included in the appendix. For higher orders, getting an analytical expression for $\psi(N)$ is difficult and in such cases, $\psi(N)$ can be obtained numerically using the corresponding SINR expression. In comparison to the approaches in [5] and [6], this method achieves better BER performance without the need for an eigenvalue calculation procedure. Also, we found that the convergence rate is faster for the MMSE-DFE compared to the MMSE-LE for which the series approximation is investigated in [4].

\section{COMPLEXity}

The polynomial expansion equalizer, involves matrix multiplication which has the same order of complexity as the matrix inversion and equal to $O\left(\left(N_{f}+N_{b}\right)^{3}\right)$. However, multiplication of the polynomial expansion with a vector in the equalizer introduces a special structure which allows us to implement it as having only vector-matrix multiplications and thus the complexity reduces to $O\left(\left(N_{f}+N_{b}\right)^{2}\right)$ [10]. As a result, in comparison with the direct matrix inversion DFE, the polynomial expansion DFE has a complexity reduction of the order of $N_{f}+N_{b}$ and an increase in complexity of the order of $N$. Since, $\mathrm{N}$ is much smaller than $N_{f}+N_{b}$ in general, the complexity savings seen in polynomial expansion DFE are significant, and it gets better as $N_{f}+N_{b}$ increases.

\section{Simulation Results and Discussions}

In our simulations, a BPSK baseband model is used. The channel is time-varying and consists of 5 taps with coefficients Gaussian distributed, having zero mean, unit variance, and following a uniform power delay profile or an exponential power delay profile. The noise is AWGN. The number of feedforward filter taps is $N_{f}=21$, while the feedback filter taps is $N_{b}=4$ taps. In this case, since $N_{b}(=4)$ is equal to the channel memory, the optimum decision delay is $\Delta=N_{f}-1$. In the Figures, $\operatorname{DFEprop}(N)$ refers to the method proposed in this paper for order $N$ where $\psi(N)$ is obtained by maximizing the SINR, DFEeig $(N)$ refers to the eigenvalue method of [6] with order $N$ i.e. $\left(\psi=\frac{2}{\lambda_{\max }+\lambda_{\min }}\right)$. Also, $\operatorname{MMSEprop}(N)$ refers to the method proposed in [4] for the MMSE-LE equalizer with order $N$. As mentioned before, the complexity involved in finding an exact analytic expression for higher order is quite high and thus the $\psi(N)$ for higher order have to be found using numerical method. This involves, finding the $\psi(N)$ that maximizes the ratio $\left(\frac{\left\|\tilde{\mathbf{w}}_{(N)}^{H} \tilde{\mathbf{h}}_{\Delta}\right\|^{2}}{\tilde{\mathbf{w}}_{(N)}^{H} \mathbf{R}_{\tilde{\mathbf{y}}_{k}} \tilde{\mathbf{w}}_{(N)}}\right)$ over a discrete set of values. Also while the methods in [5] and [6] ensure that $\psi$ lies in the range $0<\psi<\frac{2}{\lambda_{\max }}$, calculating $\psi$ using this SINR maximization method does not always guarantee this in a very few cases, so we only calculate the $\psi(N)$ that lies in the range $0<\psi<\frac{2}{\lambda_{\max }}$ and maximize the
SINR expression. The simulation results are shown in Figures $2-5$.

Fig. 2 shows BER performance comparison between DFEprop equalizer, DFEeig equalizer and direct matrix inversion MMSE-DFE. We can see that at low $\operatorname{SNR}(<10 \mathrm{~dB})$, both the proposed (DFEprop) and eigenvalue (DFEeig) equalizers perform close to the direct matrix inversion equalizer even for low order $(N=1)$. At higher SNR, a higher-order polynomial approximation is needed to achieve comparable BER performance to the direct matrix inversion based DFE. The proposed method $(\operatorname{DFEprop}(N))$ has better BER performance as compared to the existing approach (eigenvalue method, $\operatorname{DFEeig}(N))$. It is seen that when same orders are compared, $\operatorname{DFEprop}(N)$ BER performance is better than DFEeig $(N)$. Also, our proposed method at lower order $(N=5)$ is better than the eigenvalue method at higher order $(N=8)$. At high $\mathrm{SNR}(>10 \mathrm{~dB})$, DFEprop and DFEeig are not as good in BER performance for $N=1$ while there is a significant degradation for $N=0$. This is expected as $N=0$ results in just the matched filter. In general, for any order $N$, it can be seen that DFEprop achieves better BER performance than DFEeig for all SNR.

Fig. 3 shows the performance comparison of our proposed method and the MMSE-LE equalizer of [4]. It can be seen that for all SNR, the DFEprop $(N)$ is always better than the $M M S E \operatorname{rop}(N)$ equalizers in terms of BER for all orders. Also, comparing between different orders, one can observe that DFEprop (5) is better than MMSEprop (8) which is appealing as it means lower complexity of implementation due to the lower order. Our proposed method also achieves faster convergence than the MMSE-LE equalizer of [4] as it shows a good BER even for low order compared to the higher-order approximation needed for the MMSE-LE of [4]. Fig. 3 also shows that the DFEprop performs better than the MMSE-LE with direct matrix inversion even for order 5 .

Figures 2 and 3 depict simulations performed over a fading channel with uniform power delay profile. Figures 4 and 5 show the same simulations performed for fading channel with an exponential power delay profile and $3 \mathrm{~dB}$ decay over successive taps. It is noticed that for this case, the performance of the proposed equalizer gets closer to the direct matrix inversion equalizer and also at a lower order.

\section{CONCLUSION}

Based on a finite-order polynomial approximation to matrix inversion, we proposed a reduced-complexity MMSE-DFE and showed that it outperforms earlier approaches in literature. We derived a closed-form expression for the scaling parameter that is needed in the polynomial approximation for order one and described the numerical approach to find the scaling parameter for higher-order polynomial approximations. For the MMSE-DFE case, the proposed polynomial approximation performs better (in terms of performance gap from direct matrix inversion MMSE-DFE) even at lower order compared to the polynomial approximation of the MMSE-LE and also compared to earlier approaches in the literature. As expected, 


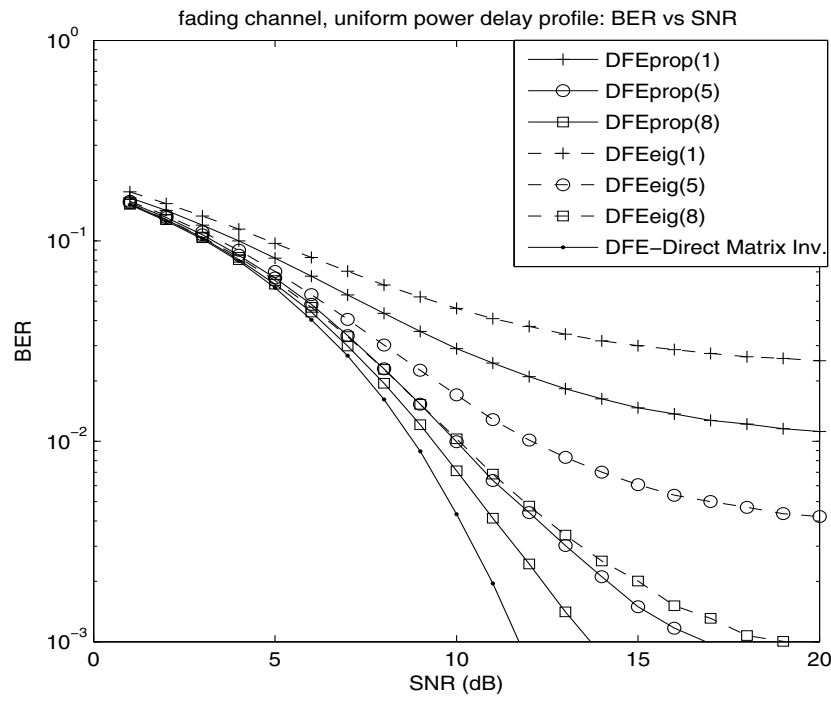

Fig. 2. Comparison between the proposed MMSE-DFE with polynomial approximation and the MMSE-DFE based on Eigenvalue method of [6] for a uniform power delay profile fading channel.

a higher-order approximation is needed at high SNR where ISI effects dominate noise effects.

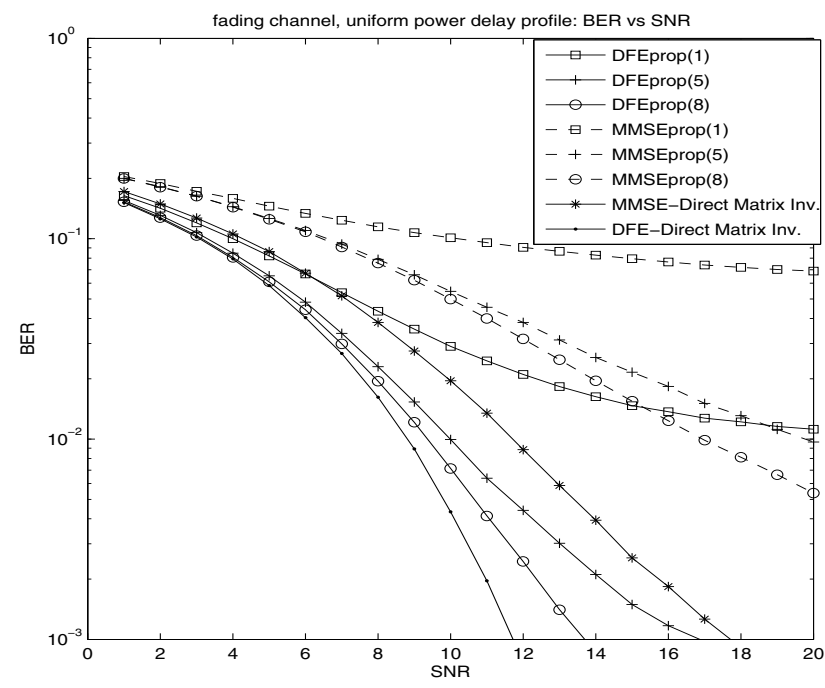

Fig. 3. Comparison between the proposed MMSE-DFE with polynomial approximation and MMSE-LE of [4] for uniform power delay profile fading channel.

\section{APPENDIX}

\section{A. Derivation for MMSE-DFE Equalizer output and SINR}

Let $\mathbf{w}=\left[w_{1}, w_{2}, \ldots, w_{N_{f}}\right]^{T}$ and $\mathbf{b}=\left[b_{1}, b_{2}, \ldots, b_{N_{b}}\right]^{T}$ be the MMSE-DFE equalizer feedforward filter and feedback filter taps. Then the equalizer output is given by

$$
\begin{aligned}
\hat{x}_{k-\Delta} & =\mathbf{w}^{H} \mathbf{y}_{k}+\mathbf{b}^{H} \mathbf{x}_{k-\Delta-N_{b}}^{k-\Delta-1} \\
& =\left[\mathbf{w}^{H} \mathbf{b}^{H}\right]\left[\mathbf{y}_{k} \mathbf{x}_{k-\Delta-N_{b}}^{k-\Delta-1}\right] \\
& =\tilde{\mathbf{w}}^{H} \tilde{\mathbf{y}}_{k}
\end{aligned}
$$

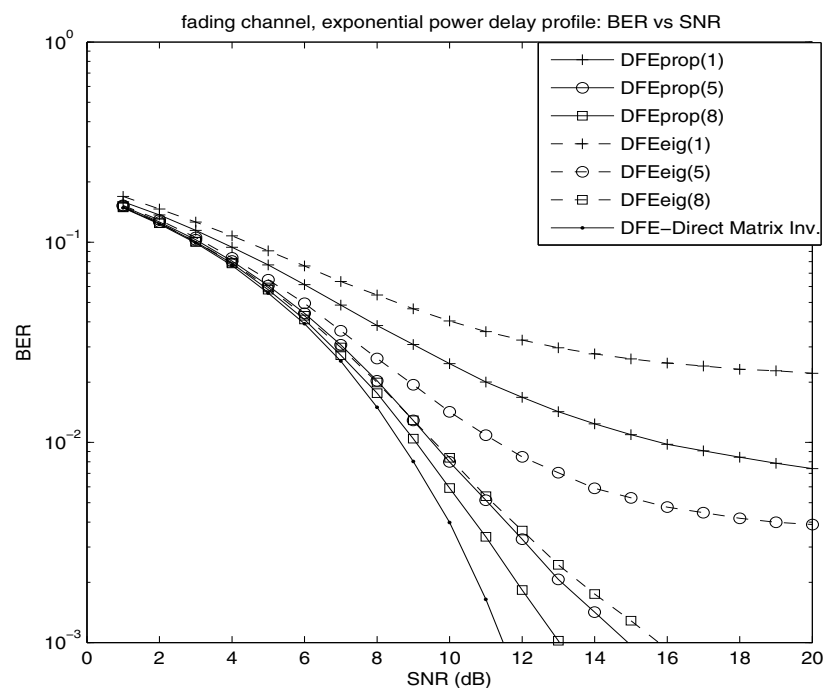

Fig. 4. Comparison between the proposed MMSE-DFE with polynomial approximation and the MMSE-DFE based on Eigenvalue method of [6] for a $3 \mathrm{~dB}$ decay exponential power delay profile fading channel.

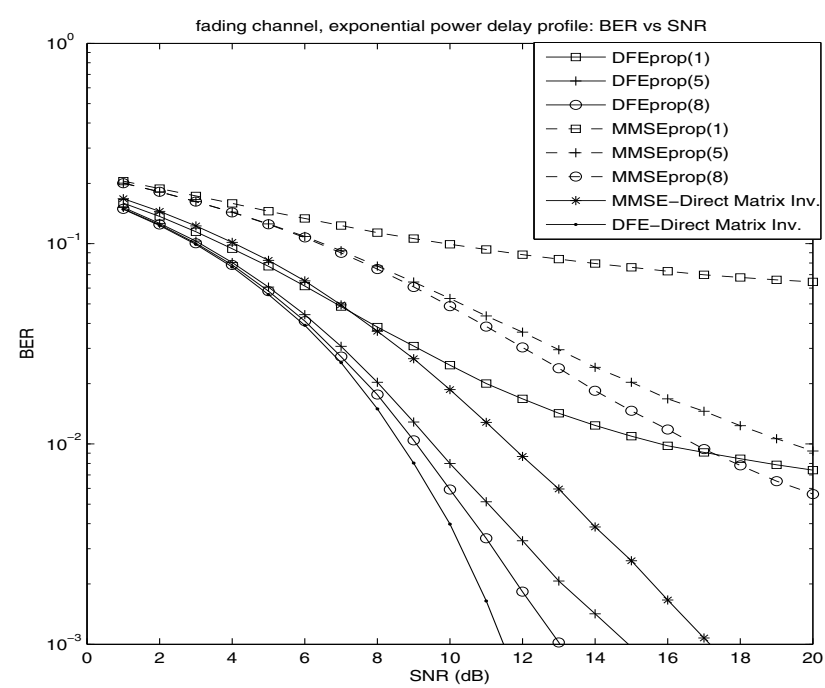

Fig. 5. Comparison between the proposed MMSE-DFE with polynomial approximation and MMSE-LE of [4] for $3 \mathrm{~dB}$ decay exponential power delay profile fading channel.

where we define

$$
\tilde{\mathbf{w}} \triangleq\left[\begin{array}{l}
\mathbf{w} \\
\mathbf{b}
\end{array}\right]
$$

and

$$
\tilde{\mathbf{y}}_{k}=\left[\begin{array}{c}
\mathbf{y}_{k} \\
\mathbf{x}_{k-\Delta-N_{b}}^{k-\Delta-1}
\end{array}\right] .
$$

Consider,

$$
\begin{aligned}
\tilde{\mathbf{w}}^{H} \tilde{\mathbf{y}}_{k} & =\tilde{\mathbf{w}}^{H}\left[\begin{array}{c}
\mathbf{y}_{k} \\
\mathbf{x}_{k-\Delta-N_{b}}^{k-\Delta-1}
\end{array}\right] \\
& =\tilde{\mathbf{w}}^{H}\left(\left[\begin{array}{c}
\mathbf{y}_{k} \\
\mathbf{0}_{N_{b} \times 1}
\end{array}\right]+\left[\begin{array}{c}
\mathbf{0}_{N_{f} \times 1} \\
\mathbf{x}_{k-\Delta-N_{b}}^{k-\Delta}
\end{array}\right]\right) \\
& =p+q
\end{aligned}
$$


where,

$$
p=\tilde{\mathbf{w}}^{H}\left[\begin{array}{c}
\mathbf{h}_{\Delta} x_{k-\Delta} \\
\mathbf{0}_{N_{b} \times 1}
\end{array}\right]
$$

is the term consisting of the useful signal, and

$$
q=\left(\left[\begin{array}{c}
\mathbf{y}_{k}-\mathbf{h}_{\Delta} \mathbf{x}_{k-\Delta} \\
\mathbf{0}_{N_{b} \times 1}
\end{array}\right]+\left[\begin{array}{c}
\mathbf{0}_{N_{f} \times 1} \\
\mathbf{x}_{k-\Delta-N_{b}}^{k-\Delta-1}
\end{array}\right]\right)
$$

is the term that doesn't contain the useful signal.

The variance of useful signal is

$$
\begin{aligned}
E\left[p^{2}\right] & =E\left[\left(\tilde{\mathbf{w}}^{H}\left(\begin{array}{c}
\mathbf{h}_{\Delta} x_{k-\Delta} \\
\mathbf{0}_{N_{b} \times 1}
\end{array}\right)\right)^{2}\right] \\
& =E\left[\left(\tilde{\mathbf{w}}^{H}\left(\begin{array}{c}
\mathbf{h}_{\Delta} \\
\mathbf{0}_{N_{b} \times 1}
\end{array}\right) x_{k-\Delta}\right)^{2}\right] \\
& =E\left[\left(\tilde{\mathbf{w}}^{H} \tilde{\mathbf{h}}_{\Delta} x_{k-\Delta}\right)^{2}\right]
\end{aligned}
$$

where

$$
\tilde{\mathbf{h}}_{\Delta}=\left(\begin{array}{c}
\mathbf{h}_{\Delta} \\
\mathbf{0}_{N_{b} \times 1}
\end{array}\right) .
$$

Therefore,

$$
E\left[p^{2}\right]=E_{x} \tilde{\mathbf{w}}^{H} \tilde{\mathbf{h}}_{\Delta} \tilde{\mathbf{h}}_{\Delta}^{H} \tilde{\mathbf{w}} .
$$

Also, the variance of the equalizer output is

$$
E\left[\hat{x}_{k-\Delta}^{2}\right]=E\left[\left(\tilde{\mathbf{w}}^{H} \tilde{\mathbf{y}}_{k}\right)^{2}\right]=\tilde{\mathbf{w}}^{H} \mathbf{R}_{\tilde{\mathbf{y}}_{k}} \tilde{\mathbf{w}}
$$

and thus,

$$
\begin{aligned}
\operatorname{SINR} & =\frac{E\left[p^{2}\right]}{E\left[\hat{x}_{k-\Delta}^{2}\right]-E\left[p^{2}\right]} \\
& =\frac{E_{x} \tilde{\mathbf{w}}^{H} \tilde{\mathbf{h}}_{\Delta} \tilde{\mathbf{h}}_{\Delta}^{H} \tilde{\mathbf{w}}}{\tilde{\mathbf{w}}^{H} \mathbf{R}_{\tilde{\mathbf{y}}_{k}} \tilde{\mathbf{w}}-E_{x} \tilde{\mathbf{w}}^{H} \tilde{\mathbf{h}}_{\Delta} \tilde{\mathbf{h}}_{\Delta}^{H} \tilde{\mathbf{w}}} \\
& =\frac{1}{\frac{\tilde{\mathbf{w}}^{H} \mathbf{R}_{\tilde{y}_{k}} \tilde{\mathbf{w}}}{E_{x} \tilde{\mathbf{w}}^{H} \tilde{\mathbf{h}}_{\Delta} \tilde{\mathbf{h}}_{\Delta}^{H} \tilde{\mathbf{w}}}-1} .
\end{aligned}
$$

For the finite impulse response MMSE-DFE, we know that [7],

$$
\begin{aligned}
& \tilde{\mathbf{w}}^{H}=E_{x}\left[\mathbf{e}_{\Delta+1}^{H} \mathbf{H}^{H} \mathbf{0}_{1 \times N_{b}}\right] \mathbf{R}_{\tilde{\mathbf{y}}_{k}}^{-1} \\
& =E_{x} \mathbf{e}_{\Delta+1}^{H} \mathbf{H}^{H}\left[\begin{array}{ll}
\mathbf{I}_{N_{f}} & \mathbf{0}_{N_{f} \times N_{b}}
\end{array}\right] \mathbf{R}_{\tilde{\mathbf{y}}_{k}}^{-1} \\
& =E_{x} \mathbf{h}_{\Delta}^{H} \tilde{\mathbf{I}}_{N_{f}} \mathbf{R}_{\tilde{\mathbf{y}}_{k}}^{-1}
\end{aligned}
$$

where

$$
\mathbf{h}_{\Delta}^{H}=\mathbf{e}_{\Delta+1}^{H} \mathbf{H}^{H}
$$

and

$$
\tilde{\mathbf{I}}_{N_{f}}=\left[\mathbf{I}_{N_{f}} \mathbf{0}_{N_{f} \times N_{b}}\right]
$$

and noting that

$$
\mathbf{h}_{\Delta}^{H} \tilde{\mathbf{I}}_{N_{f}}=\tilde{\mathbf{h}}_{\Delta}
$$

we obtain

$$
\tilde{\mathbf{w}}^{H}=E_{x} \tilde{\mathbf{h}}_{\Delta}^{H} \mathbf{R}_{\tilde{\mathbf{y}}_{k}}^{-1}
$$

Substituting (6) in (32), we get

$$
\tilde{\mathbf{w}}^{H}=E_{x} \tilde{\mathbf{h}}_{\Delta}^{H} \psi \sum_{i=0}^{\infty}\left(\mathbf{I}_{N_{f}+N_{b}}-\psi \mathbf{R}_{\tilde{\mathbf{y}}_{k}}\right)^{i} .
$$

Specifically, the first order expansion is

$$
\tilde{\mathbf{w}}^{H}(1)=E_{x} \tilde{\mathbf{h}}_{\Delta}^{H} \psi\left[\mathbf{I}_{N_{f}}+\left(\mathbf{I}_{N_{f}}-\psi(1) \mathbf{R}_{\tilde{\mathbf{y}}_{k}}\right)\right] .
$$

From (27), we see that maximizing SINR is the same as maximizing the ratio

$$
\frac{E_{x} \tilde{\mathbf{w}}^{H} \tilde{\mathbf{h}}_{\Delta} \tilde{\mathbf{h}}_{\Delta}^{H} \tilde{\mathbf{w}}}{\tilde{\mathbf{w}}^{H} \mathbf{R}_{\tilde{\mathbf{y}}_{k}} \tilde{\mathbf{w}}} .
$$

The optimal value of $\psi(1)$ is thus found by solving the following equation

$$
\frac{d}{d \psi(1)}\left(\frac{E_{x} \tilde{\mathbf{w}}^{H}(1) \tilde{\mathbf{h}}_{\Delta} \tilde{\mathbf{h}}_{\Delta}^{H} \tilde{\mathbf{w}}(1)}{\tilde{\mathbf{w}}^{H}(1) \mathbf{R}_{\tilde{\mathbf{y}}_{k}} \tilde{\mathbf{w}}(1)}\right)=0 .
$$

Substituting (34) in (36), and solving for $[\psi(1)]_{\text {opt }}$ gives the following result

$$
[\psi(1)]_{\mathrm{opt}}=2 \frac{\boldsymbol{\beta}^{H} \boldsymbol{\gamma} \boldsymbol{\beta}}{\boldsymbol{\beta}^{H} \boldsymbol{\gamma} \mathbf{R}_{\tilde{\mathbf{y}}_{k}} \boldsymbol{\beta}}
$$

where

$$
\boldsymbol{\beta}^{H}=\tilde{\mathbf{h}}_{\Delta}^{H} \mathbf{R}_{\tilde{\mathbf{y}}_{k}}
$$

and

$$
\gamma=\left\|\tilde{\mathbf{h}}_{\Delta}\right\|^{2} \mathbf{I}_{N_{f}+N_{b}}-\tilde{\mathbf{h}}_{\Delta} \tilde{\mathbf{h}}_{\Delta}^{H}
$$

\section{REFERENCES}

[1] J.G. Proakis and M. Salehi. Digital communications. McGraw-Hill Boston, 1995.

[2] N. Al-Dhahir and JM Cioffi. MMSE decision-feedback equalizers: finitelength results. IEEE Transactions on Information Theory, 41(4):961975, 1995.

[3] N. Al-Dhahir and J. M. Cioffi. Fast computation of channel-estimate based equalizers in packet data transmission. IEEE Trans. Signal Processing, 43(11):2462-2473, Nov. 1995.

[4] N. Le Josse, C. Laot, and K. Amis. Efficient series expansion for matrix inversion with application to mmse equalization. IEEE Commun. Lett., 12(1):35-37, January 2008.

[5] Z. D. Lei and T. J. Lim. Simplified polynomial-expansion linear detectors for ds-cdma systems. Electronics Letters, 34(16):1561-1563, 6 Aug. 1998.

[6] M. Mozaffaripour and R. Tafazolli. Suboptimum search algorithm in conjunction with polynomial expanded multiuser detection for uplink. In Proc. Fifth IEE International Conference on $3 G$ Mobile Communication Technologies 3G 2004, pages 103-107, 2004.

[7] John Cioffi. Class Notes for EE 379A, Chapter 3. Stanford University.

[8] H. Lutkepohl. Handbook of matrices. Chichester: Wiley, 1996.

[9] M Mozaffaripour. Multiuser Detection for Mobile CDMA Systems. $\mathrm{PhD}$ thesis, CCSR, University of Surrey, Aug. 2003.

[10] G. M. A. Sessler and F. K. Jondral. Multiuser detection for utra-tdd based on polynomial expansion. In Proc. VTC 2001 Fall Vehicular Technology Conference IEEE VTS 54th, volume 4, pages 2290-2293, 7-11 Oct. 2001. 